\section{Starlings' patterns are not spontaneous}

You incorrectly use the flocking of starlings in murmurations to illustrate the concept of 'active matter' in determining the physics of life (Nature 529, 16-18; 2016). This behaviour depends on information conveyed from individuals beyond a bird's immediate neighbours - unlike the spontaneous patterns formed in active matter from independently moving units.

In addition to information about the flight of their near neighbours, starlings use their location in the flock to adjust their murmuration behaviour (D. J. G. Pearce et al. Proc. Natl Acad. Sci. USA 111, 1042210426; 2014). The flock provides this information by projecting a 'shadow' of its surrounding density on each bird's retina. This lets the starling know whether it is on the edge of the flock or not, and safe or not from predators.

The starlings' behaviour is more akin to the idea of reflexivity (see G. Soros J. Econ. Methodol. 20, 309-329; 2013). In reflexivity, individuals act in response both to the behaviour of those immediately around them and to the sociological effect of interacting as a collective entity - in this case, the flock. Like humans, a group of starlings seems to interact by using nonlocal sociological information to shape individual behaviour. John Skoyles University College London, UK. j.skoyles@ucl.ac.uk

\section{Ancient forest: spare it from clearance}

The Białowieża Forest on the borders of Poland and Belarus is the last lowland primeval forest in Europe that is still governed by natural processes. It is now under threat from logging on an unprecedented scale, proposed by the new Polish government ostensibly to halt an outbreak of European spruce bark beetle (Ips typographus). We argue strongly for Białowieża’s preservation: it provides a much-needed ecological blueprint for restoring ancient forests, and a unique laboratory for investigating the effects of global change.

The government's proposed measures are to be included as an update to Poland's 10-year forest management plan, which in 2012 set timber extraction at 48,000 cubic metres annually. This limit was enough to meet the needs of local communities and to maintain spontaneous ecological processes.

The planned large-scale felling and salvage logging of infested trees ignores the bark beetle's keystone role in shaping the longterm dynamics and structure of forests (see B. Beudert et al. Conserv. Lett. 8, 272-281; 2015). Moreover, to contain the outbreak, it would be necessary to $\log 80 \%$ of infested trees (L. Fahse and M. Heurich Ecol. Model. 222, 1833-1846; 2011), which would mean violating the $35 \%$ of forest area that is protected. It is notable that only $57 \%$ of the stipulated harvest refers to spruce, the bark beetle's host tree species.

Przemysław Chylarecki Museum and Institute of Zoology, Polish Academy of Sciences, Warsaw, Poland.

Nuria Selva Institute of Nature Conservation, Polish Academy of Sciences, Kraków, Poland. pch@miiz.waw.pl

\section{Ancient forest: keep the logging ban}

Poland's new conservative government is planning to lift a moratorium on the logging of old-growth trees in the primeval Białowieża Forest, amid mounting opposition from the Polish Academy of Sciences, ecologists and activists.

Divided between Belarus and Poland, the 1,500-squarekilometre forest is the largest remaining part of a vast, 8,000-year-old temperate forest that blanketed the European

lowland (see Nature 455,

277-280; 2008), and is a World

Heritage Site.

The forest supports the largest population of European bison (Bison bonasus), as well as wolves, lynxes and some 120 species of breeding bird, including the lesser-spotted eagle (Clanga pomarina) and the three-toed woodpecker (Picoides tridactylus).

Poland's new environment minister, Jan Szyszko, backed by the logging lobby from Poland's state forests (see go.nature.com/ dcjp2x; in Polish), claims that the forest is "rotting away". He proposes a 33-fold increase in logging.

Although the changes do not affect the small Białowieża National Park directly, the forest's remaining old growth will disappear from the buffer zones within a couple of years and jeopardize this unique ecosystem's sustainability. Pawel Michalak Virginia Polytechnic Institute and State University, Blacksburg, USA. pawel@vbi.vt.edu

\section{Cochrane reviews expose bias too}

Cochrane is an international network of health professionals, patients and others who summarize data from research studies in systematic reviews to help users to make informed decisions about disease treatment, prevention and screening (go.nature.com/ $\mathrm{z} 5 \mathrm{w} 5 \mathrm{ah}$ ). The model we use to accomplish this in the Cochrane Eyes and Vision (CEV) US group can also expose errors of the type noted by David Allison and colleagues (Nature 530, 27-29; 2016).

In this group, clinician authors partner with a methodology 'hub' of experts who check data and apply validated methods in conducting the systematic review. These experts are trained to identify potential biases and statistical errors in reviews, and to expose what seem to be deliberate reporting biases or manipulation of outcomes.

The experts can identify errors in primary studies and draw attention to them, but cannot correct existing errors. Content experts need to collaborate with methodologists to eliminate mistakes at the outset, by designing and doing studies at a low risk of bias. Journal editors should insist that authors adhere to reporting standards. And the public must demand that health-care decisions are underpinned by conclusive scientific evidence. Kay Dickersin, Tianjing Li Johns Hopkins Bloomberg School of Public Health, Baltimore, Maryland, USA.

kdicker3@jhu.edu

Competing financial interests declared (see go.nature.com/31fu47).

\section{That's quite a collection}

Your caption under the photo of testy entomologist Harrison Dyar that accompanies the review of Marc Epstein's biography (Nature 529, 152-153; 2016) credits Dyar with having collected " 500,000 different kinds of mosquitoes over his career". Given that there are still only about 3,000 species of mosquito known worldwide today, this seems a bit of a stretch.

Dyar may have collected 500,000 specimens, but not 500,000 different kinds. Rick Borchelt College Park, Maryland, USA. rborchelt@gmail.com

\section{CORRECTION}

The Correspondence 'No myth: benefits of breast screening' (Nature 529, 283; 2016) omitted a declaration of competing interests. This has been added to the online version. 